\title{
Smart random walkers: The cost of knowing the path
}

\author{
Juan I. Perotti ${ }^{1,2, *}$ and Orlando V. Billoni ${ }^{1,2, \dagger}$ \\ ${ }^{1}$ Facultad de Matemática, Astronomía y Física, Universidad Nacional de Córdoba \\ ${ }^{2}$ Instituto de Física Enrique Gaviola (IFEG-CONICET), Ciudad Universitaria, 5000 Córdoba, Argentina
}

(Received 28 February 2012; revised manuscript received 30 May 2012; published 19 July 2012)

\begin{abstract}
In this work we study the problem of targeting signals in networks using entropy information measurements to quantify the cost of targeting. We introduce a penalization rule that imposes a restriction on the long paths and therefore focuses the signal to the target. By this scheme we go continuously from fully random walkers to walkers biased to the target. We found that the optimal degree of penalization is mainly determined by the topology of the network. By analyzing several examples, we have found that a small amount of penalization reduces considerably the typical walk length, and from this we conclude that a network can be efficiently navigated with restricted information.
\end{abstract}

DOI: 10.1103/PhysRevE.86.011120

PACS number(s): 05.40.Fb, 89.70.Hj, 89.70.Cf

\section{INTRODUCTION}

In the problem of targeted signaling or targeted navigability in a network, a message or vehicle begins a journey at a given source vertex with the intention of reaching another target vertex in the most efficient way possible. It is implicitly assumed that the message or vehicle is restricted to jumping from vertex to vertex along the edges available in the network. The many applications of this area, such as distant communication in complex systems [1] and problems related to traffic in cities [2,3], make the area an active field of research. The efficiency in solving the problem is measured in terms of a cost, which is associated with each possible path the message or vehicle can follow in its journey from the source to the target. Two main issues have to be accounted for in defining the cost of targeting: the length of the paths and the difficulty of identifying a set of convenient paths which connect the source with the target. Once the cost is defined, the efficiency in the task of targeted signaling or navigability can be improved by choosing a convenient searching strategy that minimizes the cost.

The most basic strategy for searching the target is the non-biased or fully random walker. The message or vehicle moves randomly without bias through the network with the hope that eventually it will reach the target. In this case, there is no cost associated in choosing the appropriate path to the target, so the focus is in the determination of how long it will take to the message or vehicle to reach its target. It means that the efficiency is determined by communicability between the source and the target. This problem was studied in detail by Estrada et al. [4,5], who introduced a penalty based on the lengths of the paths. Even more, this is a problem related to the first passage time and has been investigated in several paradigmatic network models [6]. It is important to stress here that the random walk strategy has to be differentiated from network sampling using random walkers [7] and from non-specific broadcasting where a signal is propagated and amplified as in the spreading of diseases, spam, or computer viruses $[8,9]$.

*perotti@famaf.unc.edu.ar

†billoni@famaf.unc.edu.ar
Other approaches deviate from a fully random walker but still dismiss the quantification of the cost of choosing the appropriated paths from the source to the target. Now the problem is finding a reasonable strategy for searching the target, and the selection of a particular strategy is driven by minimizing the length of the journey from the source to the target. Among the different strategies are self-avoiding random walks [10], intermittent random walks on lattices [11], the consideration of local topological features [12], and greedy strategies, which are used in the case of networks with spatial embedding $[13,14]$. A common feature of all the above mentioned methods is that they use knowledge about the topological structure for the design of the strategies.

A different approach to the problem consists in evaluating the difficulty of choosing the appropriate paths. The difficulty can be quantified in terms of the amount of information or knowledge required to follow these paths. In some cases the information is measured ad hoc, for example, using a fixed information cost per vertex traversed $[15,16]$. In general, the information required for choosing the right direction in the network depends on the local topological details; for instance, the information required to take the right direction grows with the number of available options. Entropy measures provide a natural way of quantifying information; in fact, this measurements have been applied successfully in complex networks before [17-21]. In particular, these methods were applied recently to quantify information in the problem of targeted signaling or navigability [2,22,23] and in the complementary problem of efficient diffusion in a network $[24,25]$. This is the approach we adopted in our work to quantify the difficulty of choosing the appropriate paths that connect the source with the target. We focus on strategies that may be adapted to any topology and in which the message or vehicle is represented by a biased random walker. These strategies will allow interpolation between a fully random walker, which uses no information to reach its destiny, and a directed walker that travels along the shortest paths using all the available information to orient itself. In this regard, there are several antecedents with strategies that interpolate to some extend the random and the biased regime [2,15,22,23,26,27].

In this work we follow the line of previous works $[15,23]$ in the sense that an information measure is used to regulate how directed the walks are. We extend the ideas of Refs. [2,22] 
where the information is measured considering only the shortest paths by allowing the usage of less information at expenses of longer walks [23]. We introduce a formalism for measuring the amount of information used by a biased random walker to reach its target. Using this formalism we develop a method which depends on one parameter that regulates how biased is the random walk. In our method the overall information is increased each time the walker performs a step, so longer walks result in larger penalization. Optimizing the walker's information forces the walker to travel along increasingly shorter paths or, equivalently, the paths are biased to the target.

The paper is organized as follow. In Sec. II we introduce the theoretical background, defining the measures of information used by the random walker in going from the source to the target. We also introduce the penalization rules used to interpolate between the random and the directed regimes; in particular, the optimal penalization is defined. In Sec. III we analyze simple examples that can be solved analytically, which are useful to understand how the method works in different topological environments, including some limiting cases. In Sec. IV we applied the method by using numerical simulations in more complex networks, such as a random network and a Barabási-Albert scale-free network model. Finally, in Sec. V final remarks, conclusions, and possible extensions to our work are discussed.

\section{THE MODEL}

Consider a non-directed network with $N$ vertices and $M$ links where a random walker jumps at a given time step from a vertex $i$ to a neighbor vertex $j$ with probability $q_{i j}$. For each vertex $i$ in the network the transition probabilities $q_{i j}$ satisfy the normalization condition,

$$
\sum_{j \in n n_{i}} q_{i j}=1
$$

where $n n_{i}$ is the set of all nearest-neighbor vertices of vertex $i$ and $q_{i i}=0$ for all $i$. This means the walker is forced to move at each time step. The amount of information given to the walker for taking an exit from a given vertex $i$ to one of its nearest neighbors is the information cost defined by [22]

$$
\ln \left(k_{i}\right)-\left[-\sum_{j \in n n_{i}} q_{i j} \ln q_{i j}\right]
$$

which is the difference between the maximum entropy in the space of events of taking one of the exits minus the entropy that the exits of vertex $i$ already have associated. Here $k_{i}$ denotes the degree of vertex $i$. Let us consider now that the walker starts its journey at a source vertex named $s$ and ends the trip at a vertex we call the target $t$; furthermore, during the journey the walker passes by the vertex $i$. We want to obtain an expression for the information needed in going from $s$ to $t$ given a distribution of probabilities $q_{i j}$. From the information cost defined above one can derive a recursive expression for the amount of information $S(i \rightarrow t)$ used by the walker in going from vertex $i$ to $t$. Accordingly this information cost is expressed as

$$
S(i \rightarrow t)=\ln \left(k_{i}\right)+\sum_{j \in n n_{i}} q_{i j} \ln q_{i j}+\sum_{j \in n n_{i}} q_{i j} S(j \rightarrow t) .
$$

Hence, with the constraint that $S(t \rightarrow t)=0$ (i.e., no information is needed by the walker once the target is reached), a set of linear equations with unknowns $\{S(i \rightarrow t)\}_{i=1, \ldots, N}$ can be defined and solved provided the probabilities $\left\{q_{i j}\right\}$ are known. A similar approach was used by Rosvall et al. [22] to quantify the amount of information needed by a walker which is restricted to walking only the shortest paths. In the case that the random walker can step back during the walk, the amount of information is [23]

$$
S_{s p}(s \rightarrow t)=-\ln \left(\sum_{\pi \in \Pi(s, t)} \frac{1}{k_{s}} \prod_{j \in \hat{\pi}} \frac{1}{k_{j}}\right),
$$

where $\Pi(s, t)$ denotes the set of all shortest paths $\pi$ between $s$ and $t$, and $\hat{\pi}$ denotes the set of interior vertices of the shortest path $\pi$.

The minimum for the information $S(s \rightarrow t)$ introduced in Eq. (3), regarding the transition probabilities $q_{i j}$, corresponds to a fully random walker with probabilities defined by

$$
q_{i j}=\frac{1}{k_{i}}, \quad \forall i, j .
$$

In this case $S(i \rightarrow t)=0$ for all $i$. As expected, in finite networks the fully random walker needs no information to reach the target, but this has the drawback of leading to very long walks on average. Since we are interested in targeted signaling, the results obtained above are of little utility. In order to fix this problem, we introduce a penalization rule that weights the paths favoring the shortest paths to the target. This penalization will modify the transition probabilities that minimize the information required to reach the target; the longer walks will be rejected and then a random walker that searches the network using these probabilities will be biased to the target.

The simplest way to introduce a penalty is by paying a cost each time the walker passes through a vertex. This information cost is not used by the walker when it is traveling the network - unlike the information associated to $q_{i j}$ - but it allows the evaluation of intrinsic properties of the paths to the target, taking into account the whole network. For instance, depending on the degree of penalty needed for reaching an optimal set of paths, one can estimate the difficulty of finding the paths in a given network. Once the penalization term is introduced in Eq. (3), it becomes

$$
\begin{aligned}
F_{\gamma}(i \rightarrow t)= & \ln \gamma+\ln \left(k_{i}\right)+\sum_{j \in n n_{i}} q_{i j} \ln q_{i j} \\
& +\sum_{j \in n n_{i}} q_{i j} F_{\gamma}(j \rightarrow t),
\end{aligned}
$$

where $\ln \gamma$, with $\gamma \geqslant 1$, is the penalization term. Now since $\gamma>1, F(s \rightarrow t)=0$ is not a minimum anymore and hence a fully random walk does not minimize the information. As shown in the next section, minimizing $F(s \rightarrow t)$ with respect to $\left\{q_{i j}\right\}$ while keeping $\gamma$ fixed leads to a biased walk, which becomes more directed to its target as $\gamma$ increases. In fact, the 
fully random walker corresponds to $\gamma=1$, and in the other extreme when $\gamma \rightarrow \infty$ the walker is forced to walk along the shortest paths. The quantity $F_{\gamma}(s \rightarrow t)$ stands for the amount of information the walker uses in going from $s$ to $t$ plus the intrinsic information related to the penalization.

To clarify the role of $\gamma$ let us introduce a quantity that will allow us to define an optimal value for the penalization. First of all, we name by $\left\{q_{i j}^{*}\right\}$ the probabilities $\left\{q_{i j}\right\}$ that minimizes $F_{\gamma}(s \rightarrow t)$ at a given fixed value of $\gamma$, and $F_{\gamma}^{*}(s \rightarrow t)$ is the function evaluated at these values, that is, the minimum. Furthermore, $S^{*}(s \rightarrow t)$ is the value of $S(s \rightarrow t)$ evaluated on $\left\{q_{i j}^{*}\right\}$. We compute the amount of information introduced by $\gamma$ as $F_{\gamma}^{*}(s \rightarrow t)-S^{*}(s \rightarrow t)$ which is related to intrinsic properties of the network, as we mentioned above. Then the relative amount of intrinsic information in going from $s$ to $t$ is

$$
R_{\gamma}(s \rightarrow t)=\frac{F_{\gamma}^{*}(s \rightarrow t)-S^{*}(s \rightarrow t)}{F_{\gamma}^{*}(s \rightarrow t)} .
$$

The quantity $R_{\gamma}(s \rightarrow t)$ lies in $(0,1]$ reaching its maximum value 1 when $\gamma \rightarrow 1$ or $\gamma \rightarrow \infty$. It has a minimum value $R^{*}(s \rightarrow t)$ at $\gamma^{*} \in(1, \infty)$, which we define as the optimal value of $\gamma$. At $\gamma^{*}$ the walker minimizes the relative amount of intrinsic information with respect to the whole information. It means that up to the minimum point, the information the walker gains above the paths to the target is preponderating. An increase of $\gamma$ above $\gamma^{*}$ certainly implies a gain of useful information, but at lower pace than the intrinsic information. Then, the value $\gamma^{*}$ gives insights about the searchability of a network in relation to its topology.

\section{SIMPLE EXAMPLES SOLVED ANALYTICALLY}

To further clarify the formal ideas introduced in the above section, let us analyze some simple examples which can be solved analytically. We named each example analyzed in order to facilitate the discussion (see Fig. 1). In addition, these examples will provide some insight to the problem of targeted delivery of information or navigation. In particular, the last two examples correspond to extreme cases in which remarkably different topological patterns prevail. On one extreme is the case where only one right path to the target exists (all the other alternative paths dead end), and on the other extreme is the
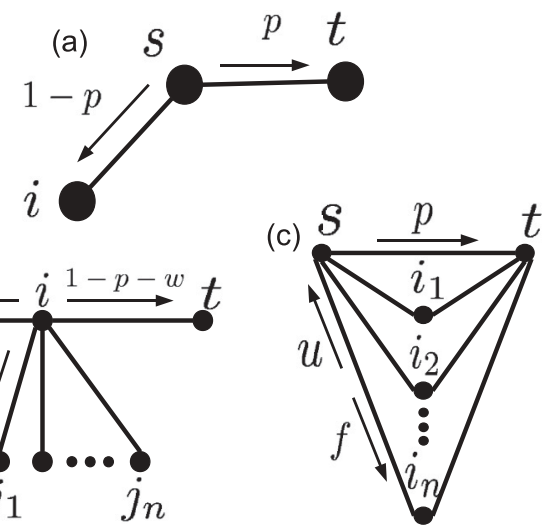

FIG. 1. Simple targeted walks in different network environments which can be solved analytically. Unique path (a), star web (b), and equivalent paths (c) (here $n f=1-p$ ). case where there are a lot of similar paths to the target-not all of them optimal-with a few short paths. The penalization scheme behaves differently in each case, serving as an indicator of which kind of topological pattern could prevail on real networks or network models.

\section{A. The unique path}

This example is outlined in Fig. 1(a). For the sake of clarity let us simplify the notation redefining $F_{\gamma}(i \rightarrow t)$ by $F_{i}$, $S(i \rightarrow t)$ by $S_{i}$, and $R_{\gamma}(i \rightarrow t)$ by $R_{i}$. In this case the set of equations (6) takes the form:

$$
\begin{aligned}
& F_{s}=\ln \gamma+\ln 2+p \ln p+(1-p) \ln (1-p)+(1-p) F_{i}, \\
& F_{i}=\ln \gamma+F_{s} .
\end{aligned}
$$

By solving the equation for $F_{s}$ and minimizing with respect to $p$ fixing $\gamma$, one obtains the following expression for the critical $p:$

$$
p^{*}=\frac{1}{2} \frac{2 \gamma^{2}-1}{\gamma^{2}} .
$$

It is easy to verify that $p^{*} \rightarrow 1 / 2$ when $\gamma \rightarrow 1$ and that $p^{*} \rightarrow 1$ when $\gamma \rightarrow \infty$; therefore one obtains the expected limiting cases. The unpenalized case, $\gamma \rightarrow 1$, corresponds to a fully random walker, and the other case, $\gamma \rightarrow \infty$, corresponds to a walker fully biased toward the shortest path. One can see that $p^{*}$ increases when $\gamma$ goes from 1 to $\infty$, indicating that the bias in the walk grows with the penalization $\gamma$; in other words, a larger penalization leads to a shorter walk. The relative amount of intrinsic information corresponding to this example,

$$
R_{s}=\frac{\left(2 \gamma^{2}+1\right) \ln \gamma}{\left(2 \gamma^{2}+1\right) \ln \gamma-\ln \gamma^{2}+\left(2 \gamma^{2}-1\right) \ln \left(\frac{2 \gamma^{2}-1}{\gamma^{2}}\right)},
$$

is plotted as a function of $\gamma$ in Fig. 2. One can see a minimum which corresponds to an optimal penalization $\gamma^{*}$. Notice that

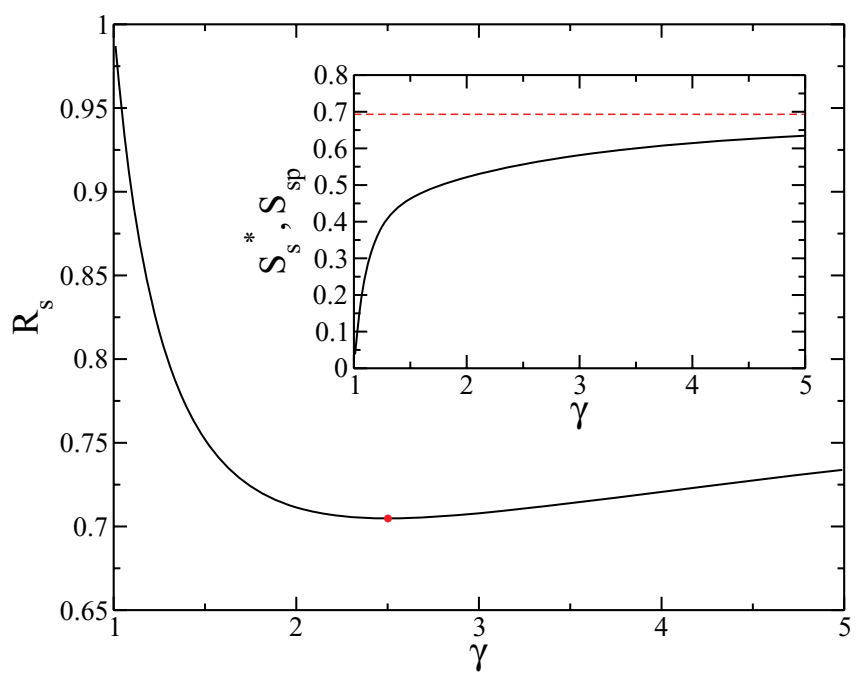

FIG. 2. (Color online) Relative amount of intrinsic information $R_{s}$ vs $\gamma$ for the unique path [see Fig. 1(a)]. The curve reaches a minimum value $\left(R_{s}^{*} \simeq 0.7\right)$ at $\gamma^{*} \simeq 2.5$ (indicated with a dot) which corresponds to the optimal degree of penalization. Inset: Optimal information $S_{s}^{*}$ (full line) and shortest path information $S_{s p}$ (horizontal red dotted line). 
$R$ is large even at $\gamma^{*}$; more than half of the total information $F^{*}$ is due to the information introduced by $\gamma$. The inset shows the information used by the walker to reach the target $S^{*}$ as function of $\gamma$. It increases as $\gamma$ increases and converges asymptotically to the case of the shortest path $S_{s p}=\ln 2$ as $\gamma \rightarrow \infty$

\section{B. The star web}

The starlike network [see Fig. 1(b)] represents the extremal case of only one direct path to the target and a large number of dead ends. At variance with the previous examples, which contain a fixed number of vertices, this example has no restrictions in the number of vertices, allowing the study of quantities that scales with the network's size. Like the previous example, this new one can be analytically solved by using the particular symmetries of this network, whatever the size of the network. Equations (6) in this case reduce to

$$
\begin{aligned}
F_{i}= & \ln \gamma+\ln (n+2)+p \ln p+(1-p-w) \ln (1-p-w), \\
& +w \ln \frac{w}{n}+p F_{s}+w F_{j}, \\
F_{j}= & \ln \gamma+F_{i}, \quad F_{s}=\ln \gamma+F_{i},
\end{aligned}
$$

where $F_{j}:=F_{j_{1}}=\cdots=F_{j_{n}}$. The critical probability of $F_{s}$ is

$$
p^{*}=\frac{1}{(n+2) \gamma^{2}} \quad \text { and } \quad w^{*}=\frac{n}{(n+2) \gamma^{2}},
$$

which satisfies the expected limiting cases: $p^{*} \rightarrow 1 /(n+2)$, $w^{*} \rightarrow n /(n+2)$ for $\gamma \rightarrow 1$, and $p^{*}, w^{*} \rightarrow 0$ for $\gamma \rightarrow \infty$. In this example, it is interesting to compute the amount of information related to shortest paths $S_{s p}$ and the optimal information $S^{*}$ at $\gamma^{*}$ as function of the number of vertices $n$. The following expression is obtained from the first, $S_{s p}(s \rightarrow$ $t)=\ln (n+2)$, while the second $\left(S^{*}\right)$ is obtained numerically. Figure 3 shows these quantities as functions of $(n+2)$ in a linear $\log$ plot. One can see that also $S^{*}$ scales logarithmically with the network's size; however, this amount of information is always smaller than the information related to the shortest path and the difference between them increases with $n$. This implies that, as far as the optimal walks defined by $\left\{q_{i j}^{*}\right\}$ are convenient, it is useful to relax the restriction of walking the shortest paths, because it is cheaper in terms of information to walk along paths which are not so short. Furthermore, the relative amount of intrinsic information $R_{s}^{*}$ decreases with the system size (see the inset of Fig. 3). It means that the walker's information about the shorter paths in the network eventually becomes predominant. This is also consistent with the decrease in the value of optimal penalization $\gamma^{*}$ as function of $n$, which is required to learn the shorter paths (see the inset). This indicates that in this topology the walker can learn efficiently the ways to the target.

\section{The equivalent paths}

This example [see Fig. 1(c)] allows us to visualize one of the main motivations to generalize the approach which measures the information considering only shortest paths $S_{s p}[22,23]$ to a measure that includes all the possible paths. Specifically, this example allows the study of a case in which the walker has many alternatives consisting of equivalent paths that are

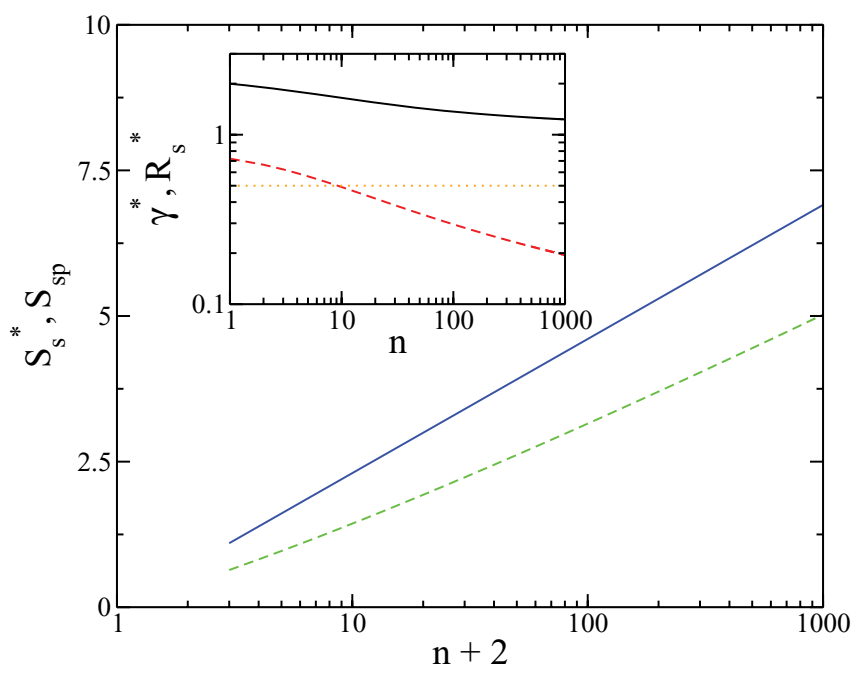

FIG. 3. (Color online) Information as function of the network's size $n$ in a linear-log plot, corresponding to the star web [see Fig. 1(b)]. Blue full line is the shortest path information $S_{s p}$, and the green dashed line is the optimal information $S_{s}^{*}$. Inset: The optimal penalization $\gamma^{*}$ (full black line). Relative amount of intrinsic information $R_{s}^{*}$ (red dashed line). Horizontal orange dotted line is the reference value for $R_{s}^{*}$ at 0.5 .

not much longer than the shortest ones. Due to its particular topology, this example can also be solved analytically for arbitrary network sizes. Accordingly, by applying Eq. (6) to this particular example, the following set of equations is generated:

$$
\begin{aligned}
F_{s}= & \ln \gamma+\ln (n+1)+p \ln p \\
& +(1-p) \ln \frac{(1-p)}{n}+(1-p) F_{i}, \\
F_{i}= & \ln \gamma+\ln 2+u \ln u+(1-u) \ln (1-u)+u F_{s},
\end{aligned}
$$

where $F_{i}:=F_{i_{1}}=\cdots=F_{i_{n}}$. As usual we solve for $F_{s}$ and minimize, leading to

$$
p^{*}=\frac{2 \gamma^{2}(n+1)-n}{(\gamma(n+1)+1)\left(n+\frac{2 \gamma^{2}(n+1)-n}{\gamma(n+1)+1}\right)}
$$

and

$$
u^{*}=1-\frac{1}{2} \frac{2 \gamma^{2}(n+1)-n}{\gamma(\gamma(n+1)+1)},
$$

which satisfy the right limits $p^{*} \rightarrow 1 /(n+1), u^{*} \rightarrow 1 / 2$, for $\gamma \rightarrow 1$ and $p^{*} \rightarrow 1, u^{*} \rightarrow 0$ for $\gamma \rightarrow \infty$. Similarly to the star $w e b$, in this example the optimal information $S^{*}$ and the shortest path information $S_{s p}$ scale logarithmically with the network's size (see Fig. 4), and also $S_{s p}>S^{*}$ but at variance with star web the difference between them remains almost constant. Here, the optimal penalization $\gamma^{*}$ grows with system size $n$ and the relative amount of intrinsic information $R^{*}$ is always predominant (see the inset of Fig. 4). These results confirm the intuitive insight that in this kind of topology it is difficult for the walker to learn the optimal walk pattern, which is related to the fact that discrimination between several similar alternatives is expensive. A comparison between this example and the previous one reveals other important differences in 


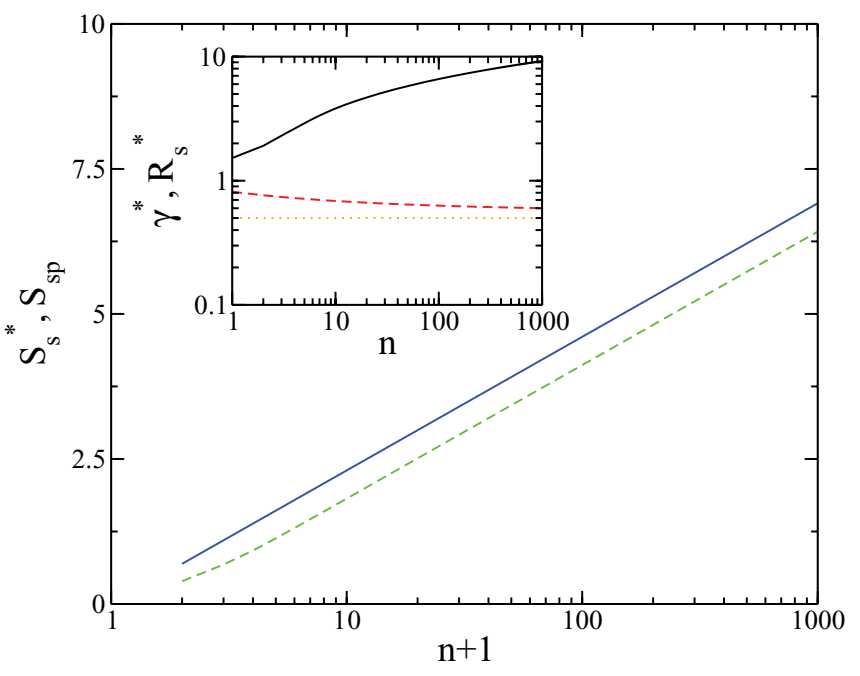

FIG. 4. (Color online) The optimal information $S_{s}^{*}$ as a function of system size $n$ (green dashed line), corresponding to the equivalent paths [see Fig. 1(c)]. This information is compared to the shortest path information $S_{s p}$ (blue full line). Both scale logarithmically with $n$ as indicated by the straight lines in the linear-log plot, and the difference $S_{s p}-S_{s}^{*}>0$ remains constant. Inset: The optimal penalization $\gamma^{*}$ (black full line) grows with $n$. The relative amount of intrinsic information dominates $R_{s}^{*}>0.5$ for all the sizes (red dashed line). The orange dotted line indicates $R_{s}^{*}=0.5$.

connection with their topologies. When dead ends prevail in a network, the optimal paths are easily achievable in an efficient way; a small penalty is enough and the relative amount of intrinsic information is not predominant. On the other hand, if the alternative paths prevail, then the optimization procedure is inefficient; a large penalty is required and the amount of intrinsic information is predominant.

\section{NUMERICAL SIMULATIONS RESULTS}

In this section we apply the ideas introduced in the previous sections to more complex network topologies. We choose two paradigmatic cases, specifically, the random and scale-free networks. Since these systems cannot be solved analytically, all the results we show here are obtained by numerical simulations. We performed an optimization procedure minimizing $F_{s}$ with respect to $\left\{q_{i j}\right\}$ for a sequence $\gamma-1=\delta, 2 \delta, 3 \delta, \ldots$, where $\delta$ is a small quantity $(\delta \in[0.005,0.05])$. This is a convenient procedure since $\left\{q_{i j}^{*}\right\}$ varies smoothly with $\gamma$. We start by using the values given by Eq. (5) as the initial guess for $\gamma=1+\delta$, and then we use the last minimum obtained for the subsequent values of $\gamma$. We perform the minimization using the implementation of the SLSQP [28,29] algorithm provided by SciPy [30] as a part of Sage Mathematics Software [31]. If $\delta$ is too large the minimization algorithm fails to converge since the initial guess is too far away from the minimum, even in small networks. The computational cost for solving the numerical problem of finding $\left\{q_{i j}^{*}\right\}$ in our approach is large. The time complexity grows as a stretched exponential of the network size, $t \sim \exp \left(a n^{1 / 2}\right)$ [32]. In practice, this prevents the problem from being solvable in large networks. At variance, other related problems [5,22] can be solved in polynomial time. The average walk length is obtained by a Monte Carlo

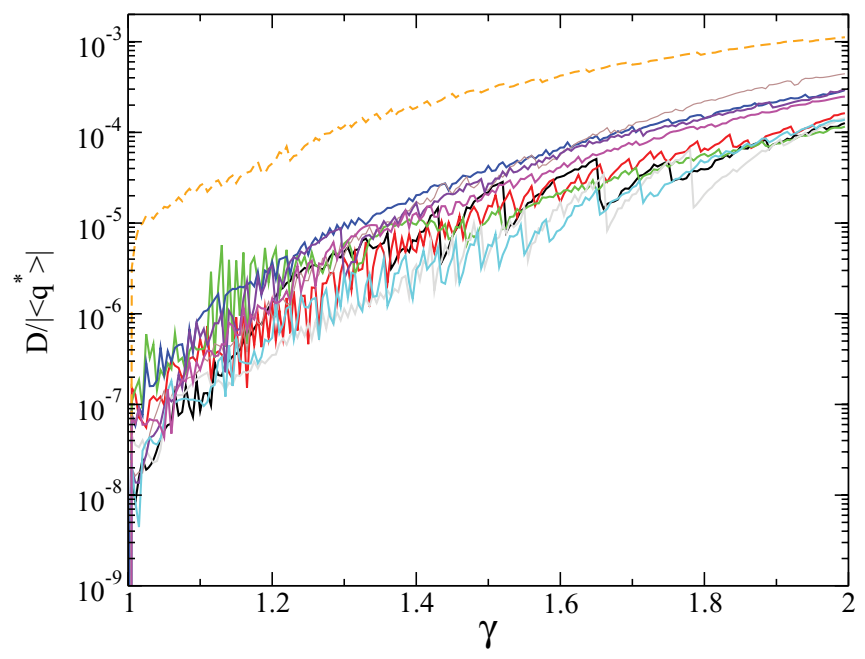

FIG. 5. (Color online) Each curve in this figure represents the ratio $D /\left\langle\vec{q}^{*}\right\rangle$ (see text) as a function of $\gamma$ for different targets $t$. One can see this ratio remains small in the whole range of $\gamma$ we explored. The largest value of the ratio is $\simeq 0.001$ (orange dotted line). The calculations were performed on a random network with $n=10$ and $\langle k\rangle=3$.

procedure using the set $\left\{q_{i j}^{*}\right\}$ for the transition probabilities. This quantity is analyzed as a function of the penalty and the shortest path length between the sources and the target.

The values of the probabilities $\left\{q_{i j}^{*}\right\}$ depend on the target vertex $t$ but are independent of the source vertex $s$ for each value of $\gamma$. We tested this analytically in the examples of Fig. 1 and numerically on a small random network. Let us define the vector $\vec{q}^{*}$ whose components are the no-null values of $\left\{q_{i j}^{*}\right\}$. In Fig. 5 we plot the ratio between the dispersion of $\vec{q}^{*}$ with respect to $s, D\left(\vec{q}^{*}\right)=1 /(n-2) \sum_{s \neq t}\left|\vec{q}^{*}(s)-\left\langle\vec{q}^{*}\right\rangle\right|^{2}$, and the norm, $\left\langle\vec{q}^{*}\right\rangle=1 /(n-1) \sum_{s \neq t} \vec{q}^{*}(s)$, as a function of $\gamma$ for different targets $t$. It is shown that this ratio is much smaller than unity, confirming the independence of $\left\{q_{i j}^{*}\right\}$ with respect to the source.

Since information is an additive quantity, the relative amount of intrinsic information for the overall network can be defined, given target $t$. It considers all the possible sources and hence the paths to the target $t$. Consequently we have

$$
R_{\gamma}(t)=\frac{\sum_{i \neq t} F_{\gamma}(i \rightarrow t)-S(i \rightarrow t)}{\sum_{i \neq t} F_{\gamma}(i \rightarrow t)},
$$

and then the overall optimal penalization $\gamma_{t}^{*}$ associated to the target $t$ can be obtained from this expression. For the sake of brevity let us omit the reference to $t$, so we refer to $R_{\gamma}(t)$ by $R_{\gamma}$ and its optimal version $R^{*}(t)$ by $R^{*}$.

\section{A. Random networks}

Let us first analyze the case of a random network. All the calculations in this section were performed using a network of $N=100$ vertices with an average degree $\langle k\rangle=3$ and using a target chosen at random. Care was taken to obtain a random network that consists of only one connected component. Here we show results corresponding to a single realization of the target and the network, since similar results were obtained 


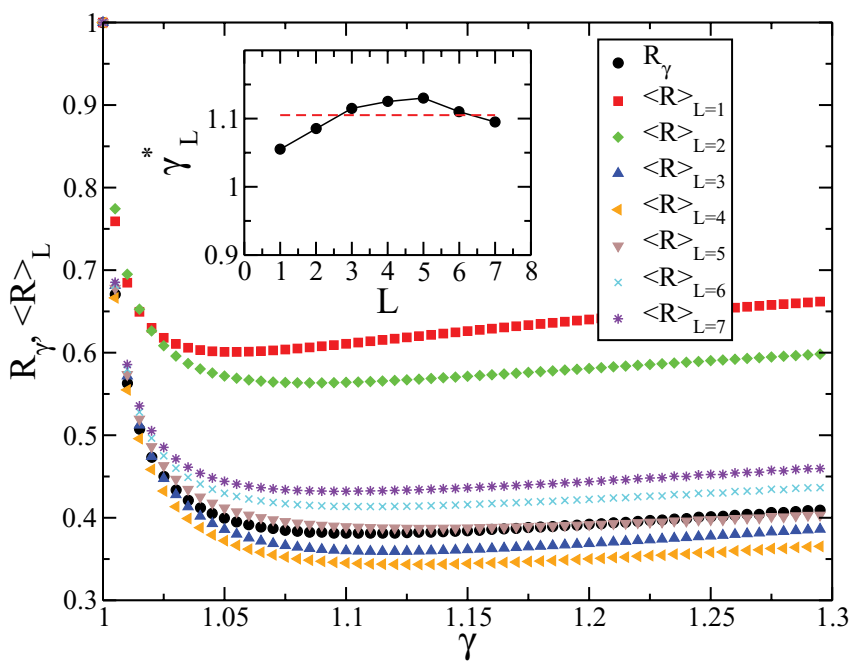

FIG. 6. (Color online) In black full circles the relative amount of intrinsic information $R_{\gamma}$ associated with a randomly chosen vertex $t$ [Eq. (16)]. It shows the typical behavior with a minimum at $\gamma_{t}^{*} \simeq$ 1.105. The other curves correspond to the relative amount of intrinsic information averaged over all the sources at a fixed distance $L=$ $1,2, \ldots$ from $t$. Inset: The minimum of $\langle R\rangle_{L} ; \gamma_{L}^{*}$, depends on $L$, but is the same order as $\gamma_{t}^{*}$ (red dotted line).

using different realizations. Figure 6 shows $R_{\gamma}$ as function of $\gamma$ obtained for this network. From this curve we obtained the optimal overall penalization $\gamma_{t}^{*} \simeq 1$.105. In this figure we also show $\langle R\rangle_{L}$, which is the relative information $R_{s}$ averaged over the sources that are at a fixed distance $L$ from the target. We obtained from these curves the optimal penalization $\gamma_{L}^{*}$; the dependence of $\gamma_{L}^{*}$ on $L$ is shown in the inset. We observe that $\gamma_{L}^{*}$ varies with $L$ but in every case $\gamma_{L}^{*}$ is of the same order of magnitude that $\gamma_{t}^{*}$.

In order to analyze the role of the penalty in the restricted walks toward the target, we analyze typical walk lengths as function of $\gamma$. We first obtain the transition probabilities associated with the target $t$, for a given $\gamma$, and then using these probabilities we implement a Monte Carlo process to obtain a set of trajectories corresponding to random walkers which are biased to the target. The random walkers start their journies at every possible source available in the network. When calculating the average of these trajectories we obtain the average walk length $\langle w l\rangle$. In Fig. 7 we plot $\langle w l\rangle$ corresponding to the same realization of the network and target we used to obtain the results of Fig. 6. When $\gamma$ approaches its minimal physical value $\gamma=1$ the walk is fully random and the average walk length between all the possible sources and the fixed target is $\langle w l\rangle \simeq 260$, which is at least an order of magnitude larger than the typical distance between vertices. Then as $\gamma$ is increased the average walk length decreases drastically. We denote by $\langle L\rangle_{t}$ the average of all the shortest paths that reach the target (note that in this calculation all sources are included). At the optimal penalization $\gamma_{t}^{*}$ the difference between the average walk length and the average shortest paths $\langle L\rangle_{t}$ is nearly two times $\langle L\rangle_{t}$; that is, the average walk length is of the same order of magnitude as the average of the shortest paths. We also computed the average walk length $\left\langle w l^{*}\right\rangle_{L}$ at the optimal penalization $\gamma_{t}^{*}$ that corresponds to averages in

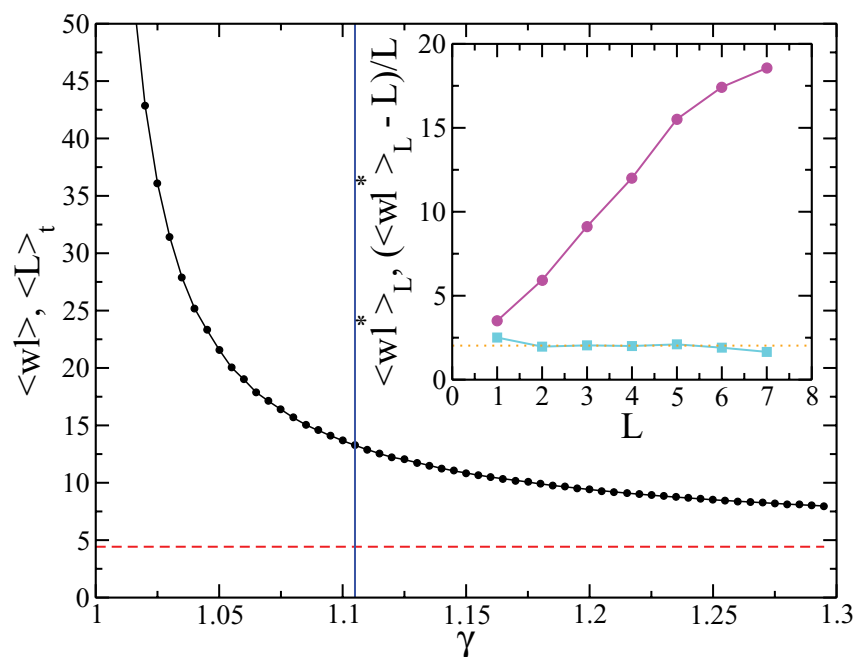

FIG. 7. (Color online) In black full circles the walk length $\langle w l\rangle$ averaged over different sources for a fixed target $t$ as a function of the penalization $\gamma$. The red dashed line indicates average shortest path length $\langle L\rangle_{t}$ to $t$. The vertical blue full line indicates the optimal value of the penalization $\gamma_{t}^{*}$. Inset: The magenta full circles correspond to the average walk length at optimality $\left\langle w l^{*}\right\rangle_{L}$ as a function of the distance to $t$. Cyan full squares represents the relative difference $\left(\left\langle w l^{*}\right\rangle_{L}-L\right) / L$ and the orange dotted line indicates the value 2 .

trajectories restricted to start at sources that are at a distance $L$ from the target. We plotted this quantity as function $L$ in inset of Fig. 7. The average walk length $\left\langle w l^{*}\right\rangle_{L}$ increases linearly with the shortest path length $L$, but the relative excess $\left(\left\langle w l^{*}\right\rangle_{L}-L\right) / L$ is almost constant, taking a value close to 2 (see inset of Fig. 7). In order to explore the dependency of the walker's information $S$ on the penalty, we plot in Fig. 8 the

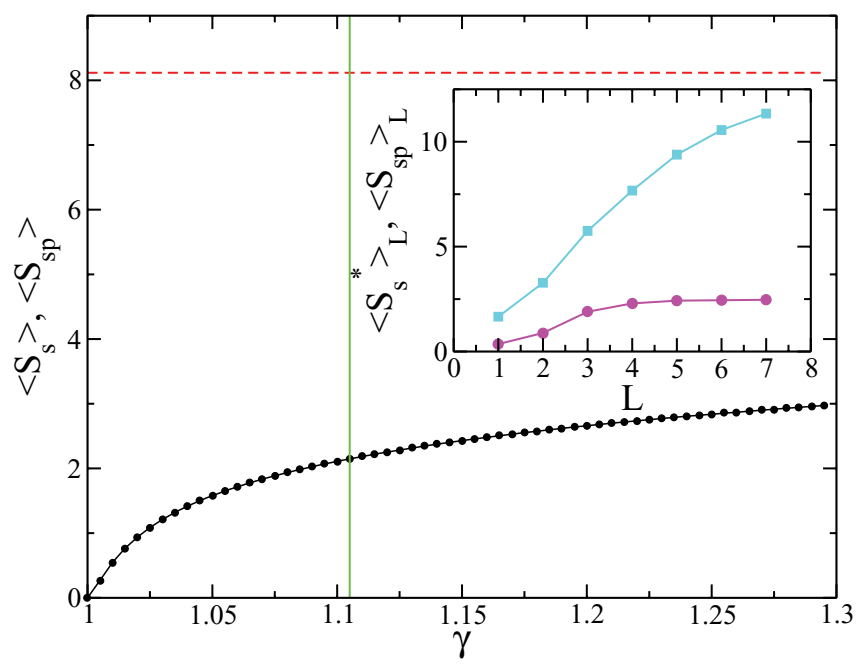

FIG. 8. (Color online) Walker's information $\left\langle S_{s}\right\rangle$ averaged over all the sources as function of the penalty $\gamma$ (black full circles). The horizontal red dashed line indicates the average shortest path information $\left\langle S_{s p}\right\rangle$. Vertical green full line indicates the optimal penalization $\gamma_{t}^{*}$. Inset: Average walker's information $\left\langle S_{s}^{*}\right\rangle_{L}$ at optimal penalty $\gamma_{t}^{*}$ as function of the distance $L$ (magenta full circles) and shortest path information $\left\langle S_{s p}\right\rangle_{L}$ (cyan full squares). 
average, $\left\langle S_{s}\right\rangle$, over all the sources as a function of $\gamma$. It can be seen that $\left\langle S_{s}\right\rangle$ grows with $\gamma$ but it is always much smaller than the averaged shortest path information $\left\langle S_{s p}\right\rangle$, in particular at the overall optimal penalization $\gamma_{t}^{*}$. In the present approach the walker uses less information than in the shortest path approach $S_{s p}$ but there is a price to pay for it; the path length to the target $\langle w l\rangle$ is longer than the shortest path $L$. In addition, according to the current approach the amount of information the walker learns on average $\left\langle S_{s}^{*}\right\rangle_{L}$ does not depend on $L$ (inset in Fig. 8) as long as $L$ is greater than 3 . One can think that there is a distance horizon $(L=3)$ that defines two regimes. At short distances the walker can improve its information about the paths to the target as the distance grows, whereas for targets far away the sources, the amount of information cannot be improved. In other words, the searchability at short distance is favored $[23,33]$.

\section{B. Scale-free networks}

Scale-free networks are characterized by a power law distribution in the connectivity of the vertices, and even small networks shows the presence of highly connected vertices when compared to the mean value of their connectivity. Therefore, although in our case the size of the network is small, a scale-free topology will allow us to study how the different quantities are affected by the vertex's degree. We performed the calculations on a Barabási-Albert network model [34] with $N=100$ vertices and $\langle k\rangle \simeq 4$. Figure 9 shows that the walk length $\left\langle w l^{*}\right\rangle$ and the shortest path length $\langle L\rangle$ decrease with target's degree and the decrease of the former is more pronounced. The hubs can be found easily having a walk length much closer to the shortest paths, whereas in poorly connected vertices significantly longer walks are required. According to the present approach, the hubs are favored, regarding both

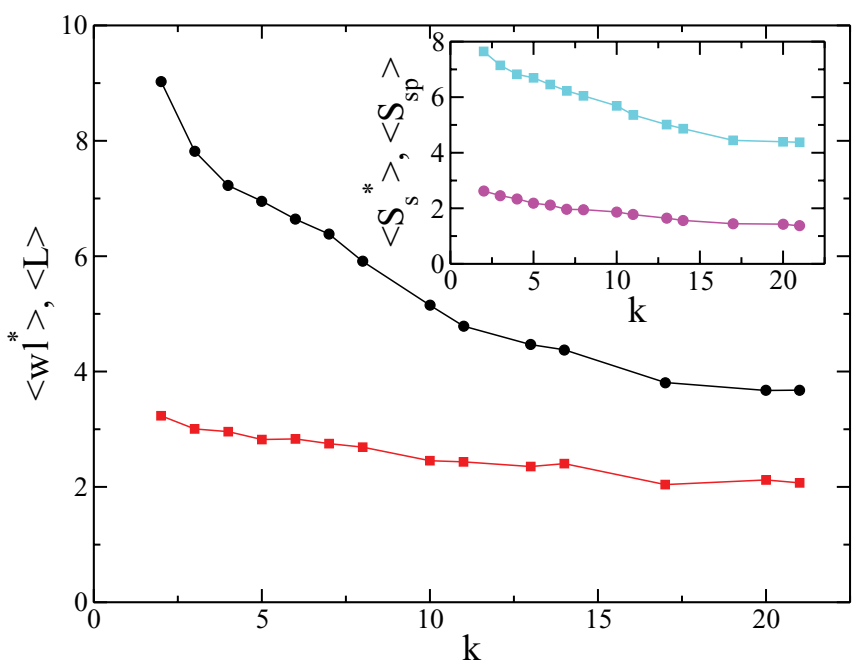

FIG. 9. (Color online) Average walk length $\left\langle w l^{*}\right\rangle$ at optimal penalization $\gamma_{t}^{*}$ (black full circles) and the average shortest path length $\langle L\rangle$ (red full squares) as a function of the target degree $k$. Both quantities decrease as the degree increases. Inset: Average walker's information $\left\langle S_{s}^{*}\right\rangle$ at optimal penalty $\gamma_{t}^{*}$ (magenta full circles) and the shortest path information $\left\langle S_{s p}\right\rangle$ (cyan full squares) as a function of the target degree.

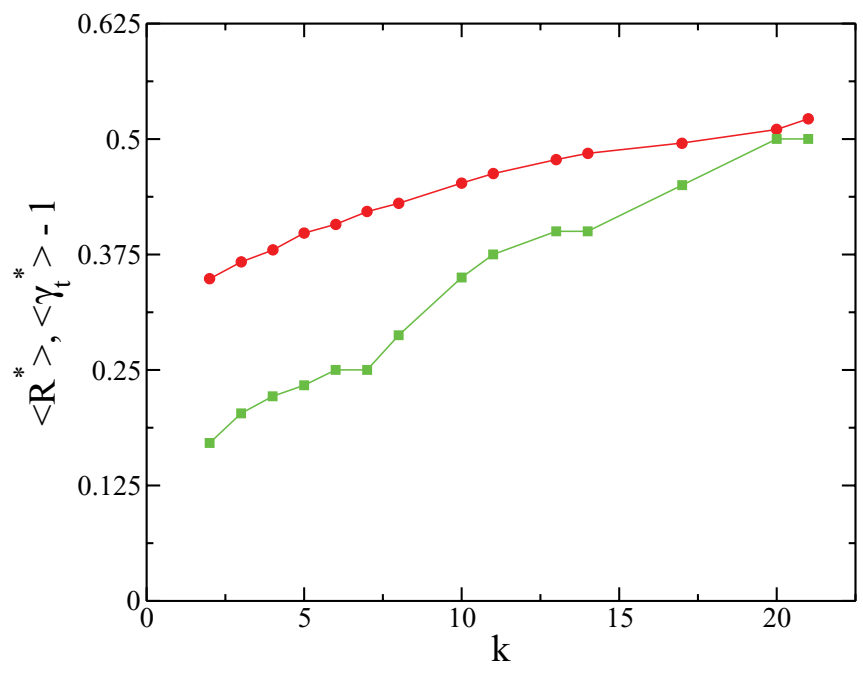

FIG. 10. (Color online) Average relative amount of intrinsic information $\left\langle R^{*}\right\rangle$ (red full circles) and average of the overall optimal penalization $\left\langle\gamma_{t}^{*}\right\rangle$ as a function of the target degree $k$ (green full squares).

the number of steps and the information that is needed, as far as the optimal condition is easily achievable. As in the case of the random network, in the scale-free networks the walker's information $\left\langle S_{s}^{*}\right\rangle$ is significantly smaller than the shortest path information $\left\langle S_{s p}\right\rangle$ (see inset of Fig. 9). Both quantities decrease with the target's degree, confirming that highly connected vertices are easier to find. Since the shortest path information $\left\langle S_{s p}\right\rangle$ varies more steeply than the walker's information $\left\langle S_{s}^{*}\right\rangle$ with the target's degree $k$, then it follows that $\left\langle S_{s p}\right\rangle$ is more sensitive to the topological details than $\left\langle S_{s}^{*}\right\rangle$. Finally, Fig. 10 shows that the optimal penalization $\gamma_{t}^{*}$ and the relative amount of intrinsic information $R^{*}$ grows with the target's degree. This implies that it is more expensive to find the optimal walking pattern in highly connected targets. As in scale-free networks, finite-size effects may be very important, especially for such small networks as used here. We repeated the numerical calculations of Figs. 9 and 10 ten times [32] for extentions of these calculations. In order to check if the trend found is not due to the particular structure of the Barabási-Albert network model, we randomized the networks using the algorithm of Maslov-Sneppen [35]. All the samples show the same tendency as the original calculation.

The scale-free networks should approach the star web as the degree exponent increases. In order to test this hypothesis we generate scale-free tailed networks ${ }^{1}$ [32] with varying

\footnotetext{
${ }^{1}$ We follow the idea in Ref. [23]. A sample of the degree distribution $P(k) \sim\left(k_{0}+k\right)^{-\alpha}$ is obtained using the inverse transform sampling method. Then, a network is generated from this sample using the Havel-Hakimi algorithm [36] and randomized using the MaslovSneppen algorithm [35]. Since the star web has $\langle k\rangle=2$ we generate networks with connectivities near this value. This can be done in scale-free networks when $\alpha \simeq 1.16$, but for larger values of $\alpha$ larger values of $\langle k\rangle$ are required in order to obtain nonfragmented networks. We choose $\langle k\rangle(\alpha=1.16)=2,\langle k\rangle(\alpha=3)=2.5$, and $\langle k\rangle(\alpha=4)=$ 2.75 by properly setting the value of $k_{0}$.
} 

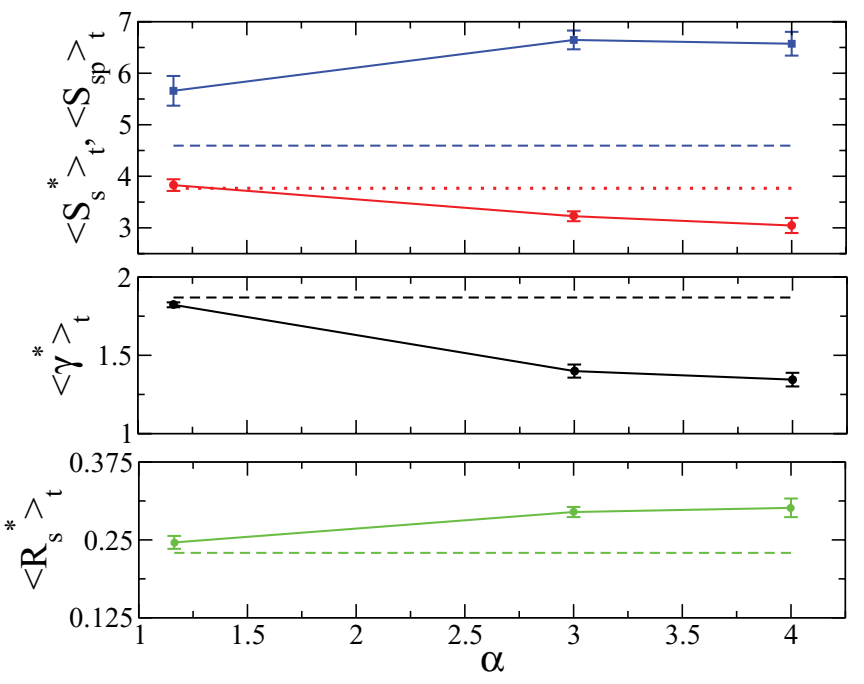

FIG. 11. (Color online) Comparison of different magnitudes of fat-tailed networks with degree distribution $P(k) \sim\left(k_{0}+k\right)^{-\alpha}$ (symbols connected with full lines) against the star web (horizontal dotted and dashed lines) as function of $\alpha$. Top: Optimal information $S^{*}($ hub $\rightarrow t$ ) (red circles and dotted line) and shortest path information $S_{s p}($ hub $\rightarrow t$ ) (blue squares and dashed line) averaged over the targets. Center: Optimal penalty $\gamma^{*}$ averaged over the targets. Bottom: Optimal intrinsic information $R_{s}^{*}$ averaged over the targets.

exponent $-\alpha$. In Fig. 11 different quantities measured in the scale-free network are plotted as function of $\alpha$; as reference we also include the values corresponding to the star web. The magnitudes for the scale-free networks approach the values for the star web as $\alpha$ decreases. In particular, the optimal information $\left\langle S_{s}^{*}\right\rangle_{t}$ (shortest path information $\left\langle S_{s p}\right\rangle_{t}$ ) is smaller (greater) for the $\alpha$-dependent networks than for the star web. These two facts imply that the difference between the shortest path information $S_{s p}$ and the optimal information $S_{s}^{*}$ decreases as the hetereogeneity of the network grows. In particular, the difference diminishes significantly only when $\alpha<3$, which is the range where the network heterogeneity is relevant as the variance $\operatorname{var}(k)$ diverges for infinite networks. The shortest path information for the $\alpha$-dependent networks is larger than that of the star web because inevitably, multiple steps along highly connected nodes are required in the walk from the source to the target.

\section{DISCUSSION AND CONCLUSIONS}

In this work we have introduced an approach for measuring the amount of information used by a biased random walker that moves to a target. In this framework, we extend the ideas of Rosvall et al. [22] because we consider not only the shortest paths but all the possible paths to the target. Based in this approach we propose a penalization rule, which depends on one parameter and that bias a random walker to the target, provided the walker can use as little information as possible. The basic idea was that each step that the walker takes is penalized; hence, this leads to an overall penalty that tends to reduce the walk lengths. Our approach is consistent since the two main quantities that determine the cost associated with the task of targeted signaling are counterbalanced: a shortening of the walk length through penalization implies an increase of the information required and vice versa. At this point, it is important to stress that in this scheme the penalization operates globally, limiting the overall available information, unlike other approaches [23] in which the information at each vertex is limited. This has the advantage of overcoming the undesired effect of affecting mostly the highly connected vertices, provided the vertex's degrees are taken into account $[15,23]$. We also introduce the idea of intrinsic information, in order to define an optimal penalization. We have shown through some network models that in practice a small amount of penalization is enough to drastically reduce the typical walk length, and then a network can be efficiently navigated with restricted information. On the other hand, once the optimal penalization is reached it is highly expensive to further reduce the typical walk length; in particular, an infinite penalization is required to restrict the path lengths to the shortest ones. The typical walk length in a random network was analyzed and compared to the corresponding shortest path at the optimal penalization. It is found that the difference between these lengths grows linearly with the shortest path length. This is connected with a trade-off at which the amount of information does not increase with the length of the shortest paths. In addition, from the trend of $\langle S\rangle_{L}$ a distance horizon can be identified which defines a range of efficient searchability. It is worth stressing that the existence of an information horizon has been previouly reported in the literature. In the contexts of targeted signaling by Trusina et al. [37], and in the complementary subject of efficient network diffusion by Sinatra et al. [25] where an optimal diffusion process is attainable with local limited information.

The ideas introduced in this paper were applied to undirected and unweighted networks. However, in the study of traffic in cities, directed and weighted networks are needed since streets have different capacities and directions. The extension of the formalism to include directed networks is straightforward, but care must be taken to ensure that each vertex is accessible from each other vertex; otherwise the analysis has to be restricted to each strongly connected components of the network. Also the penalty scheme may be generalized to be vertex dependent. In the case of the dual representation of network's cities where vertices are streets and edges are road intersections [2], the traffic congestion on each street can be used to regulate the amount of penalization in order to avoid a traffic jam. Although the proposed formalism requires the solution of an optimization problem which has a large computational cost, the algorithm is especially suited for a parallel implementation since each target $t$ can be treated separately.

\section{ACKNOWLEDGMENTS}

The authors thank Sergio Cannas, Daniel Stariolo and Pablo Serra for useful discussions. This work was partially supported by grants from CONICET (Argentina), Agencia Córdoba Ciencia (Argentina), SeCyT, Universidad Nacional de Córdoba (Argentina). 
[1] K. Sneppen, A. Trusina, and M. Rosvall, Europhys. Lett. 69, 853 (2005).

[2] M. Rosvall, A. Trusina, P. Minnhagen, and K. Sneppen, Phys. Rev. Lett. 94, 028701 (2005).

[3] S. Scellato, L. Fortuna, M. Frasca, J. Gómez-Gardeñes, and V. Latora, Eur. Phys. J. B 73, 303 (2010).

[4] E. Estrada and N. Hatano, Phys. Rev. E 77, 036111 (2008).

[5] E. Estrada, N. Hatano, and M. Benzi, Phys. Rep. 514, 89 (2012).

[6] J. D. Noh and H. Rieger, Phys. Rev. Lett. 92, 118701 (2004).

[7] L. F. Costa and G. Travieso, Phys. Rev. E 75, 016102 (2007).

[8] Y. Moreno and A. Vázquez, Eur. Phys. J. B 31, 265 (2003).

[9] J. Balthrop, S. Forrest, M. E. J. Newman, and M. M. Williamson, Science 304, 527 (2004).

[10] S.-J. Yang, Phys. Rev. E 71, 016107 (2005).

[11] G. Oshanin, K. Lindenberg, and H. Wio, J. Phys. A 42, 434008 (2009).

[12] L. A. Adamic, R. M. Lukose, A. R. Puniyani, and B. A. Huberman, Phys. Rev. E 64, 046135 (2001).

[13] J. M. Kleinberg, Nature (London) 406, 845 (2000).

[14] A. P. S. de Moura, A. E. Motter, and C. Grebogi, Phys. Rev. E 68, 036106 (2003)

[15] D. O. Cajueiro, Phys. Rev. E 79, 046103 (2009).

[16] D. O. Cajueiro, Phys. A (Amsterdam, Neth.) 389, 1945 (2010).

[17] R. Solé and S. Valverde, in Complex Networks, Lecture Notes in Physics, Vol. 650, edited by E. Ben-Naim, H. Frauenfelder, and Z. Toroczkai (Springer, Berlin, 2004), pp. 189-207.

[18] L. Demetrius and T. Manke, Phys. A (Amsterdam, Neth.) 346, 682 (2005).

[19] G. Bianconi, Phys. Rev. E 79, 036114 (2009).
[20] Y. Lin, K. C. Desouza, and S. Roy, Appl. Math. Comput. 216, 2824 (2010)

[21] E. Capobianco, J. Comput. Sci. 2, 144 (2011).

[22] M. Rosvall, A. Grönlund, P. Minnhagen, and K. Sneppen, Phys. Rev. E 72, 046117 (2005).

[23] M. Rosvall, P. Minnhagen, and K. Sneppen, Phys. Rev. E 71, 066111 (2005).

[24] Z. Burda, J. Duda, J. M. Luck, and B. Waclaw, Phys. Rev. Lett. 102, 160602 (2009).

[25] R. Sinatra, J. Gómez-Gardeñes, R. Lambiotte, V. Nicosia, and V. Latora, Phys. Rev. E 83, 030103 (2011).

[26] V. Sood and P. Grassberger, Phys. Rev. Lett. 99, 098701 (2007).

[27] A. Fronczak and P. Fronczak, Phys. Rev. E 80, 016107 (2009).

[28] D. Kraft, Technical Report DFVLR-FB 88-28, Institut für Dynamik der Flugsysteme, Oberpfaffenhofen, 1988 (unpublished).

[29] D. Kraft, ACM Trans. Math. Softw. 20, 262 (1994).

[30] E. Jones, T. Oliphant, P. Peterson et al., SciPy: Open Source Scientific Tools for Python (2001-), http://www.scipy.org/.

[31] W. Stein et al., Sage Mathematics Software (Version 4.6.2), Sage Development Team (2011), http://www.sagemath.org.

[32] See Supplemental Material at http://link.aps.org/supplemental/ 10.1103/PhysRevE.86.011120 for more details.

[33] M. Rosvall and K. Sneppen, Int. J. Bifurcation Chaos Appl. Sci. Eng. 17, 2509 (2007).

[34] A.-L. Barabási and R. Albert, Science 286, 509 (1999).

[35] S. Maslov and K. Sneppen, Science 296, 910 (2002).

[36] G. Chartrand and L. Lesniak, Graphs and Digraphs, 3rd ed. (Chapman and Hall, New York, 1996).

[37] A. Trusina, M. Rosvall, and K. Sneppen, Phys. Rev. Lett. 94, 238701 (2005). 